\title{
Sign conjugacy classes of the alternating groups Lucia Morotti
}

\begin{abstract}
A conjugacy class $C$ of a finite group $G$ is a sign conjugacy class if every irreducible character of $G$ takes value 0,1 or -1 on $C$. In this paper we classify the sign conjugacy classes of alternating groups.
\end{abstract}

\section{Introduction}

We will begin this paper by giving the definition of sign conjugacy class for an arbitrary finite group.

Definition 1.1. Let $G$ be a finite group. A conjugacy class of $G$ is a sign conjugacy class of $G$ if every irreducible character of $G$ takes values 0, 1 or -1 on $C$.

In [4] Olsson considered sign conjugacy classes of $S_{n}$ in order to answer a question from Isaacs and Navarro for $S_{n}$, using the following property of sign conjugacy classes:

Theorem 1.2. Let $C$ be a sign conjugacy class of a finite group $G$ and define $\chi^{+}$and $\chi^{-}$by

$$
\chi^{+}:=\sum_{\substack{\chi \in \operatorname{Irr}(G): \\ \chi(C)>0}} \chi \quad \text { and } \quad \chi^{-}:=\sum_{\substack{\chi \in \operatorname{Irr}(G): \\ \chi(C)<0}} \chi .
$$

Then $\chi^{+}$and $\chi^{-}$are characters of $G$ differing only on $C$.

In [4] Olsson also formulated a conjecture about sign conjugacy classes of $S_{n}$ which was proven in [2]. In this paper we will classify sign conjugacy classes of $A_{n}$, proving that they are closely related to those of $S_{n}$, as would be to expect due to the relationship of irreducible characters of $S_{n}$ and $A_{n}$.

As we will be working with alternating (and symmetric) groups, we will refer to partitions instead of conjugacy classes. This leads to the following two definitions:

Definition 1.3. A partition $\gamma$ is a sign partition if it is the cycle partition of a sign conjugacy class of $S_{n}$.

If $\gamma$ is an even partition then $\gamma$ is an $A_{n}$-sign partition if it is the cycle partition of a sign conjugacy class of $A_{n}$. 
For example (3) is a sign partition which is not an $A_{n}$-sign partition, while $(2,2)$ is an $A_{n}$-sign partition which is not a sign partition. In general however $A_{n}$-sign partitions are almost always sign partitions and almost all even sign partitions are $A_{n}$ sign partitions, as will be seen from Theorem 1.6 and Theorem 1.3 of [2].

For $\gamma$ consisting of odd distinct parts it is clear that $C_{\gamma,+}$ is a sign conjugacy class of $A_{n}$ if and only if $C_{\gamma,-}$ is also such a conjugacy class (later it will be proved that no such conjugacy class is a sign conjugacy class). In particular any conjugacy class of $A_{n}$ is a sign conjugacy class exactly when its cycle partition is an $A_{n}$-sign partition.

In order to describe $A_{n}$-sign partitions we will need the two following sets of partitions:

Definition 1.4. We define Sign to be the subsets of partitions consisting of all partitions $\left(\gamma_{1}, \ldots, \gamma_{r}\right)$ for which there exists an $s, 0 \leq s \leq r$ such that the following holds:

- $\gamma_{i}>\gamma_{i+1}+\ldots+\gamma_{r}$ for $1 \leq i \leq s$,

- $\left(\gamma_{s+1}, \ldots, \gamma_{r}\right)$ is one of the following partitions:

- (), $(1,1),(3,2,1,1)$ or $(5,3,2,1)$,

$-(a, a-1,1)$ with $a \geq 2$,

$-(a, a-1,2,1)$ with $a \geq 4$,

$-(a, a-1,3,1)$ with $a \geq 5$.

Definition 1.5. We define $\overline{\operatorname{Sign}}$ by:

$$
\begin{aligned}
\overline{\text { Sign }:=} & \{(1,1,1),(2,2),(2,2,1),(5,4,3,2,1)\} \\
& \cup\{(a, a-1,4,1): a \geq 6\} \\
& \cup\{(a, a-3,2,1,1): a=6 \text { or } a \geq 8\} \\
& \cup\{(a, a-5,3,2,1): 9 \leq 4 \leq 10 \text { or } a \geq 12\} \\
& \cup\{(a, b, a-b+1): b+1 \leq a \leq 2 b-2\} \\
& \cup\{(a, b, a-b-1,1): b+2 \leq a \leq 2 b\} .
\end{aligned}
$$

In [2] (Theorem 1.3) it was proven that a partition $\gamma$ is a sign partition if and only if $\gamma \in$ Sign, proving a conjecture of Olsson from [4]. In this paper we will prove the following characterization of $A_{n}$-sign conjugacy classes.

Theorem 1.6. Let $\gamma$ be a partition of $n \geq 2$. Then $\gamma$ is an $A_{n}$-sign partition if and only if

$\gamma \in(\operatorname{Sign} \cup \overline{\text { Sign }}) \cap\{$ even partitions not consisting of odd distinct parts $\}$. 
In order to prove Theorem 1.6 we will use the following lemmas.

Lemma 1.7. Let $\gamma$ be an $A_{n}$-sign partition. If $\gamma \notin\{(1,1,1),(2,2),(2,2,1)\}$ then $\gamma$ does not have repeated parts, except possibly for the part 1, which may have multiplicity 2.

Lemma 1.8. Let $\alpha=\left(\alpha_{1}, \ldots, \alpha_{h}\right)$ be a partition with $h \geq 3$. Assume that $\alpha_{1}>\alpha_{2}$, that $\alpha \notin$ Sign and that $\left(\alpha_{2}, \ldots, \alpha_{h}\right) \in$ Sign. Then if $\alpha \neq$ $(5,4,3,2,1)$ we can find a partition $\beta$ of $|\alpha|$ such that $\chi_{\alpha}^{\beta} \notin\{0, \pm 1\}$ and $h_{2,1}^{\beta}=\alpha_{1}$.

If $\alpha \notin \overline{\text { Sign }}$ we can choose $\beta$ to be not self conjugate.

These two lemmas will allow us to prove in Section 3 that, if $\gamma=$ $\left(\gamma_{1}, \ldots, \gamma_{r}\right)$ is an $A_{n}$-sign partition, then $\left(\gamma_{i}, \ldots, \gamma_{r}\right) \in$ Sign for $i \geq 2$ and $\gamma \in$ Sign $\cup \overline{\text { Sign }}$, by otherwise constructing partitions $\delta$ which are not self conjugate and which satisfy $\chi_{\gamma}^{\delta} \notin\{0, \pm 1\}$, contradicting then the assumption that $\gamma$ is an $A_{n}$-partition. In Section 4 we will prove that partitions consisting of odd distinct parts are not $A_{n}$-sign partitions (by simply looking at the characters of $A_{n}$ indexed by the corresponding self conjugate partition), proving then one direction of Theorem 1.6. The other direction will be proved in Section 5. Through all of the following $\gamma$ will be a partition of $n \geq 2$.

From Theorem 1.3 of 2 and from Theorem 1.6 we also easily obtain that if $\chi_{\gamma}^{\delta} \notin\{0, \pm 1\}$ and $\gamma \in \overline{\text { Sign }}$ does not consist of distinct odd parts, then $\delta$ is self conjugate. This can be proved also if $\gamma \in \overline{\operatorname{Sign}}$ consist of distinct odd parts, using arguments similar to those from Section 5 .

Proofs of results about irreducible characters of $S_{n}$ and $A_{n}$ and about weights, cores and quotients of partitions can be found in [1] and [3].

\section{Proof of Lemmas 1.7 and 1.8}

We will now prove Lemmas 1.7 and 1.8. To do this we will use results from [2] and [4]. Since most of the partitions considered there are of the form $\left(a, b, 1^{c}\right)$ we will first classify in the next lemma which such partitions are self conjugate. In the following if $\beta$ is a partition we will write $\beta^{\prime}$ for its conjugate.

Lemma 2.1. A partition $\left(a, b, 1^{c}\right)$ of $n \geq 2$ is self conjugate if and only if $c=a-2$ and $b \in\{1,2\}$. In particular if $\left(a, b, 1^{c}\right)$ is self conjugate then $b+c \in\{a-1, a\}$. 
Proof. Let $\beta:=\left(a, b, 1^{c}\right)$. If $b=0$ clearly $\beta$ is not self conjugate, as $a=n \geq 2$. So assume that $b \geq 1$. From $\beta_{2}^{\prime} \leq 2$ it follows that if $\beta$ is self conjugate then $b \in\{1,2\}$. As clearly $\left(a, 1^{c+1}\right)$ and $\left(a, 2,1^{c}\right)$ are self conjugate if and only if $c=a-2$ the lemma follows.

We will now prove Lemma 1.7.

Proof of Lemma 1.7. Assume that $\gamma$ has a repeated part (with multiplicity at least 3 if this part is 1$)$.

If $\gamma \notin\{(1,1,1),(2,2)\}$ and $\gamma$ is not of the form $(a, a, 1)$ for $a \geq 4$ or of the form $\left(\delta_{1}, \ldots, \delta_{t}, \mu_{1}, \ldots, \mu_{v}\right)$ with $\delta_{t}>\mu_{1}$ and

$$
\mu \in\left\{\left(2^{m}, 1\right),\left(2^{m}, 1^{2}\right),\left(3^{m}, 2,1\right),\left(3^{m}, 2,1^{2}\right),\left(3^{m}, 1\right),\left(3^{m}, 1^{2}\right): m \geq 2\right\},
$$

we can find from the proof of Lemma 6 and Theorem 7 of [4] a partition $\beta$ with $\chi_{\gamma}^{\beta} \neq\{0, \pm 1\}$ which, from Lemma 2.1, is not self conjugate. It then easily follows that $\gamma$ is not an $A_{n}$-sign partition. In the not covered cases let

$$
\beta= \begin{cases}(a+1, a), & \gamma=(a, a, 1), \\ \left(n-2,1^{2}\right), & \mu=\left(2^{m}, 1\right) \text { or }\left(2^{m}, 1^{2}\right), \\ (n-3,3), & \mu=\left(3^{m}, 2,1\right),\left(3^{m}, 2,1^{2}\right) \text { or }\left(3^{m}, 1\right), \\ \left(n-3,1^{3}\right), & \mu=\left(3^{m}, 1^{2}\right),\end{cases}
$$

where $n=|\gamma|$. It can be easily checked using Lemma 2.1 that the above partitions are not self-adjoint unless $\gamma=(2,2,1)$. Also $\chi_{\gamma}^{\beta} \notin\{0, \pm 1\}$, for example

$$
\chi_{\left(\delta_{1}, \ldots, \delta_{t}, 2^{m}, 1\right)}^{\left(n-2,1^{2}\right)}=\chi_{\left(2^{m}, 1\right)}^{\left(2 m-1,1^{2}\right)}=-(m-1) \chi_{(2,1)}^{(3)}+\chi_{(2,1)}^{\left(1^{3}\right)}=-m .
$$

The lemma then follows.

We will now prove Lemma 1.8, Most of the work will be in proving that if $\alpha \notin \overline{\text { Sign }}$ then the partitions $\beta$ constructed in the proof of Theorem 1.6 of [2] are not self-adjoint. This is always the case apart for $\alpha=\left(\alpha_{2}+2 a-\right.$ $\left.1, \alpha_{2}, a, a-1,1\right)$ with $\alpha_{2}>2 a$ and $a \geq 4$, which will be treated separately.

Proof of Lemma 1.8. We will divide the proof of the lemma in the following cases: 1) $\alpha=\left(\alpha_{2}+2 a-1, \alpha_{2}, a, a-1,1\right)$ with $\alpha_{2}>2 a$ and $\left.a \geq 4,2\right)$ all other cases. Case 2) will be divided in subcases corresponding to the different cases of the proof of Theorem 1.6 of [2].

1) For $\alpha=\left(\alpha_{2}+2 a-1, \alpha_{2}, a, a-1,1\right)$ with $\alpha_{2}>2 a$ and $a \geq 4$ let $\beta:=\left(\alpha_{2}+2 a, 3,1^{\alpha_{2}+2 a-4}\right)$. Since $a \geq 4$ we have that $\beta$ is a partition. Also $h_{2,1}^{\beta}=\alpha_{2}+2 a-1=\alpha_{1}$ and $\beta$ is not self-adjoint from Lemma 2.1. As

$$
h_{1,3}^{\beta}=\alpha_{2}+2 a+2-3=\alpha_{1},
$$


as $a \geq 4$ so that

$$
\alpha_{2}+2 a-4>\alpha_{2}
$$

and as any partition of $\alpha_{2}+2 a$ has at most one $\alpha_{2}$-hooks since $\alpha_{2}>2 a$, we have

$$
\begin{aligned}
\chi_{\alpha}^{\beta} & \left.=(-1)^{\alpha_{2}+2 a-4} \chi_{\left(\alpha_{2}, a, a-1,1\right)}^{\left(\alpha_{2}+2 a\right)}-\chi_{\left(\alpha_{2}, a, a-1,1\right)}^{\left(2,2,1^{\alpha}+2 a-4\right.}\right) \\
& =(-1)^{\alpha_{2}+2 a-4}-(-1)^{\alpha_{2}-1} \chi_{(a, a-1,1)}^{\left(2,2,1^{2 a-4}\right)} \\
& =(-1)^{\alpha_{2}+2 a-4}-(-1)^{\alpha_{2}-1+a-1} \chi_{(a-1,1)}^{\left(2,2,1^{a-4}\right)} \\
& =(-1)^{\alpha_{2}+2 a-4}-(-1)^{\alpha_{2}-1+a-1+a-3} \chi_{(1)}^{(1)} \\
& =(-1)^{\alpha_{2}} 2 .
\end{aligned}
$$

So the lemma holds in this case.

2) In each of the following cases $\beta$ is as constructed in [2] for the corresponding case. Notation used here is as in [2].

- For

$$
\begin{aligned}
\left(\alpha_{2}, \ldots, \alpha_{h}\right) \in \quad & (1,1),(3,2,1,1),(5,3,2,1)\} \\
& \cup\{(a, a-1,1): 2 \leq a \leq 4\} \\
& \cup\{(a, a-1,2,1): 4 \leq a \leq 8\} \\
& \cup\{(a, a-1,3,1): 5 \leq a \leq 10\}
\end{aligned}
$$

the lemma can be checked by looking at each single case separately (they are finitely many since $\alpha_{1} \leq \alpha_{2}+\ldots+\alpha_{h}$ ).

- For

$$
\begin{aligned}
\left(\alpha_{2}, \ldots, \alpha_{h}\right) \in \quad & \{(a, a-1,1): a \geq 5\} \\
& \cup\{(a, a-1,2,1): a \geq 9\} \\
& \cup\{(a, a-1,3,1): a \geq 11\}
\end{aligned}
$$

we can apply Lemma 2.1 to results from Section 2 of [2] and, as $\alpha_{1}>$ $\alpha_{2}=a \geq 5$, obtain that if $\beta$ is self conjugate then $\alpha$ is either $(2 a, a, a-$ $1,1)$ or $(a+1, a, a-1,1)$, as in all other cases $\beta=\left(|\alpha|-\alpha_{1}, b, 1^{\alpha_{1}-b}\right)$ with $|\alpha|-\alpha_{1} \geq \alpha_{1}+2$ or $b \geq 4$.

If $\alpha=(a+1, a, a-1,1)$ then $\beta=(a-1, a-1, a-1,4)$ is self conjugate if and only if $a=5$, that is $\alpha=(6,5,4,1) \in \overline{\text { Sign. }}$.

If $\alpha=(2 a, a, a-1,1)=(2 a, a, 2 a-a-1,1)$. As $a \geq 5$ we have that $\alpha \in \overline{\text { Sign. }}$. 
- For $\alpha$ as in Theorems 3.1 or 3.2 of 2 we have from Lemma 2.1 that if $\beta$ is self conjugate then $x=1$ and $|\alpha|-\alpha_{1}=\alpha_{1}$. From $x=1$ we have that $k=h$ and $\alpha_{h}=1$. From $|\alpha|-\alpha_{1}=\alpha_{1}$ we have that $\alpha_{1}=\alpha_{2}+\ldots+\alpha_{h}$, that is $\alpha_{1}-\alpha_{2}=\alpha_{3}+\ldots+\alpha_{h}$, so that $k=4$. So $\alpha=\left(\alpha_{1}, \alpha_{2}, \alpha_{1}-\alpha_{2}-1,1\right)$. From $\alpha_{1}-\alpha_{2}>\alpha_{h}=1$ and $\alpha_{2}>\alpha_{1}-\alpha_{2}-1$ it follows that $\alpha \in \overline{\text { Sign }}$ when $\beta$ is self-adjoint.

- For $\alpha$ as in Theorem 3.3 of [2], $\beta$ is not self-adjoint from Lemma 2.1, as

$$
\beta_{2}=\alpha_{1}-c=\alpha_{2}+\alpha_{k-1}+\ldots+\alpha_{h}-c>\alpha_{2}>2 .
$$

- For $\alpha$ as in Theorem 3.4 of [2] we have that if $\beta$ is self conjugate then $k=4$ and $\left(\alpha_{k-1}, \ldots, \alpha_{h}\right)=(a, a-1,1)$, as in all other cases $\alpha_{1} \neq|\alpha|-\alpha_{1}-1$. Here $\alpha_{1}=\alpha_{2}+2 a-1$ and $\alpha_{2}>2 a$. The case $a \geq 4$ has already been considered in 1). For $a=2$ or $a=3$ instead we have $\alpha \in \overline{\text { Sign }}$.

- For $\alpha$ as in Theorems 3.5, 3.9 and 3.11 of [2], $\beta$ is not self-adjoint from Lemma 2.1, as $\alpha_{1}>2$.

- For $\alpha$ as in Theorems 3.6, 3.7 and 3.10 of [2] the lemma follows again from Lemma 2.1, as $\alpha_{2}>1$.

- For $\alpha$ as in Theorem 3.8 of [2], if $\beta$ is self-adjoint then $\alpha_{1}=|\alpha|-\alpha_{1}+1$. By assumption $|\alpha|-\alpha_{1} \geq \alpha_{2}+\alpha_{h}>\alpha_{1}$, so that if $\beta$ is self-adjoint then $h=3$ and $\alpha=\left(\alpha_{1}, \alpha_{2}, \alpha_{1}-\alpha_{2}+1\right)$. As $\alpha_{1}>\alpha_{2}>\alpha_{1}-\alpha_{2}+1$, so that $\alpha_{2}+1 \leq \alpha_{1} \leq 2 \alpha_{2}-2$, it follows that $\alpha \in \overline{\operatorname{Sign}}$ when $\beta$ is not self conjugate.

- For $\alpha$ as in Theorem 3.12 of [2] we have that $\beta_{2}=\alpha_{3} \geq 4=\beta_{2}^{\prime}$. In particular $\beta$ is not self conjugate for $\alpha_{3} \geq 5$. For $\alpha_{3}=4$ then $\alpha=\left(\alpha_{1}, \alpha_{1}-1,4,1\right) \in \overline{\operatorname{Sign}}$, as $\alpha_{1}-1>4$, so that $\alpha_{1} \geq 6$.

- For $\alpha$ as in Theorem 3.13 of [2] we have that $\alpha_{3}>\alpha_{4} \geq \alpha_{h-1}=2$ as $h \geq 5$ and then $\alpha_{1}>\alpha_{2}>\alpha_{3}+\alpha_{4} \geq 5$. In particular $\alpha_{1} \geq 7$ and then $\beta_{2}^{\prime}=4<\alpha_{1}-2=\beta_{2}$ so that $\beta$ is not self conjugate.

- For $\alpha$ as in Theorem 3.14 of [2] if $\beta$ is self-adjoint then $\alpha_{h-1}=3$ and

$$
|\alpha|-\alpha_{1}-\alpha_{h-1}+1=\alpha_{1}-\alpha_{h-1}-1+\alpha_{h-1}-3+3 .
$$

So $\alpha_{2}+\ldots+\alpha_{h}=\alpha_{1}+1$, which from the assumptions is equivalent to $\alpha_{3}+\ldots+\alpha_{h-1}=1$. Always from the assumption this would give

$$
3<\alpha_{3}+\ldots+\alpha_{h-1}=1
$$


leading to a contradiction. So $\beta$ is not self-adjoint.

\section{$3 \quad A_{n}$-sign partitions are elements of Sign $\cup \overline{\operatorname{Sign}}$}

Let $\gamma$ be an $A_{n}$-sign partition. If $r \leq 2$ then clearly $\gamma \in \operatorname{Sign} \cup \overline{\operatorname{Sign}}$ from Lemma 1.7 (if $r=2$ and $\gamma_{1}=\gamma_{2}$ then $\gamma \in\{(1,1),(2,2)\}$ ).

So assume now that $r \geq 3$. From Lemma 1.7 we have that $\left(\gamma_{r-1}, \gamma_{r}\right) \in$ Sign. Also either $\gamma \in\{(1,1,1),(2,2,1)\}$ and then $\gamma \in \overline{\text { Sign }}$ or $\gamma_{i}>\gamma_{i+1}$ for $1 \leq i \leq r-2$. Assume now that for some $2 \leq i \leq r-2$ we have $\left(\gamma_{i+1}, \ldots, \gamma_{r}\right) \in \operatorname{Sign}$ and $\left(\gamma_{i}, \ldots, \gamma_{r}\right) \notin$ Sign. Then $\gamma_{i}>\gamma_{i+1}$ and so, if $\left(\gamma_{i}, \ldots, \gamma_{r}\right) \neq(5,4,3,2,1)$, we can find $\beta$ with $\chi_{\left(\gamma_{i}, \ldots, \gamma_{r}\right)}^{\beta} \notin\{0, \pm 1\}$ and $h_{2,1}^{\beta}=$ $\gamma_{i}$. From $2 \leq i \leq r-2$ it follows $r \geq 4$, so that $\gamma \notin\{(1,1,1),(2,2,1)\}$ and then $\gamma_{j}>\gamma_{i}$ for $j<i$. Let

$$
\delta:=\left(\beta_{1}+\gamma+1+\ldots+\gamma_{i-1}, \beta_{2}, \beta_{3}, \ldots\right) .
$$

As $h_{2,1}^{\delta}=h_{2,1}^{\beta}=\gamma_{i}$ and $i \geq 2$ we have

$$
\delta_{1}^{\prime} \leq h_{2,1}^{\delta}+1=\gamma_{i}+1<1+\gamma_{1} \leq \delta_{1},
$$

so that $\delta$ is not self conjugate. From

$$
\chi_{\gamma}^{\delta}=\chi_{\left(\gamma_{i}, \ldots, \gamma_{r}\right)}^{\beta} \notin\{0, \pm 1\},
$$

we then have a contradiction to $\gamma$ being an $A_{n}$-sign partition.

Assume now that $\left(\gamma_{i}, \ldots, \gamma_{r}\right)=(5,4,3,2,1)$. If $\gamma_{i-1} \geq 7$ let

$$
\delta:=\left(4+\gamma_{1}+\ldots+\gamma_{i-1}, 4,4,3\right) .
$$

In this case $\delta_{1}^{\prime}=4<11 \leq \delta_{1}$, so that also in this case $\delta$ is not self conjugate. As $\gamma_{i-1} \geq 7$,

$$
\chi_{\gamma}^{\delta}=\chi_{(5,4,3,2,1)}^{(4,4,4,3)}=-2
$$

and then also in this case we have a contradiction. If instead $\gamma_{i-1}=6$ let

$$
\delta:=\left(15+\gamma_{1}+\ldots+\gamma_{i-2}, 2,1,1,1,1\right) .
$$

Here too $\delta$ is not self conjugate and

$$
\chi_{\gamma}^{\delta}=\chi_{(6,5,4,3,2,1)}^{(15,2,1,1,1,1)}=2,
$$

again leading to a contradiction.

By induction $\left(\gamma_{2}, \ldots, \gamma_{r}\right) \in \operatorname{Sign}$. Assume now that $\gamma \notin \operatorname{Sign} \cup \overline{\operatorname{Sign}}$. Then from Lemma 1.8 (as $\gamma \notin\{(1,1,1),(2,2,1)\}$, so that $\gamma_{1}>\gamma_{2}$ from Lemma 1.7 in this case) there exists $\beta$ not self conjugate with $\chi_{\gamma}^{\beta} \notin\{0, \pm 1\}$, again leading to a contradiction.

In particular if $\gamma$ is an $A_{n}$-sign partition then $\gamma \in \operatorname{Sign} \cup \overline{\operatorname{Sign}}$. 


\section{Partitions consisting of odd distinct parts are not $A_{n}$-sign partitions}

Let $\gamma$ consists of odd distinct parts and let $\lambda$ be the self conjugate partition with diagonal hook lengths equal to the parts of $\gamma$. Then

$$
\chi_{\gamma,+}^{\lambda, \pm}=\frac{\epsilon \pm \sqrt{\epsilon \gamma_{1} \cdots \gamma_{r}}}{2}
$$

with $\epsilon \in\{ \pm 1\}$.

If $\epsilon=-1$ clearly $\chi_{\gamma, \pm}^{\lambda, \pm} \notin\{0, \pm 1\}$.

Assume now that $\epsilon=1$. From $\gamma$ being a partition of $n \geq 2$ it follows that $\gamma_{1} \cdots \gamma_{r} \geq 2$. In particular $\chi_{\gamma,+}^{\lambda,+}>1$.

Similarly $\chi_{\gamma,-}^{\lambda, \pm}$ are not both in $\{0, \pm 1\}$ and then $\gamma$ is not an $A_{n}$-sign partition. Together with Section 3 this prove that if $\gamma$ is an $A_{n}$-sign partition then

$\gamma \in(\operatorname{Sign} \cup \overline{\operatorname{Sign}}) \cap\{$ even partitions not consisting of odd distinct parts $\}$.

\section{Elements of Sign $\cup \overline{\operatorname{Sign}}$ are $A_{n}$-sign partitions}

We will now prove that if

$\gamma \in(\operatorname{Sign} \cup \overline{\operatorname{Sign}}) \cap\{$ even partitions not consisting of odd distinct parts $\}$

then $\gamma$ is an $A_{n}$-sign partition. If $\gamma \in$ Sign this is easily proved in the next theorem.

Theorem 5.1. Let $\gamma \in$ Sign be an even partition not consisting of odd distinct parts. Then $\gamma$ is an $A_{n}$-sign partition.

Proof. From Theorem 1.3 of [2] we have that $\gamma$ is a sign partition. So $\chi_{\gamma}^{\beta} \in$ $\{0, \pm 1\}$ for every $\beta \vdash n$. In particular $\chi_{\gamma} \in\{0, \pm 1 / 2, \pm 1\}$ and then also $\chi_{\gamma} \in\{0, \pm 1\}$ for every irreducible character $\chi$ of $A_{n}$, that is $\gamma$ is an $A_{n}$-sign partition.

For $\gamma \in \overline{\text { Sign }}$ the proof is more complicated. It can be checked that $(1,1,1),(2,2),(2,2,1),(5,4,3,2,1),(6,5,4,1),(6,3,2,1,1),(9,4,3,2,1)$ and $(10,5,3,2,1)$ are $A_{n}$-sign partitions by looking at the corresponding characters tables. For the other elements of Sign we will use the following lemmas.

Lemma 5.2. If $\gamma \in \overline{\text { Sign }}$ does not consist of odd distinct parts, then $\gamma$ is an $A_{n}$-sign partition if and only if $\chi_{\gamma}^{\beta} \in\{0, \pm 1\}$ for every $\beta \vdash n$ not self conjugate with at least two $\gamma_{1}$-hooks. 


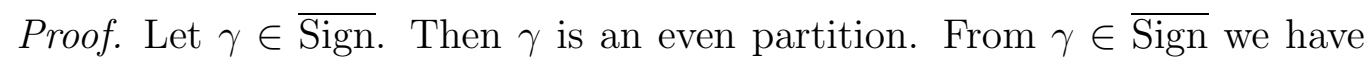
that $\left(\gamma_{2}, \ldots, \gamma_{r}\right) \in$ Sign and that $|\gamma| \leq 3 \gamma_{1}$. In particular any $\beta \vdash n$ has at most $3 \gamma_{1}$-hooks. As $\left(\gamma_{2}, \ldots, \gamma_{r}\right) \in$ Sign and then it is a sign partition from Theorem 1.3 of [2], it follows that $\chi_{\gamma}^{\beta} \in\{0, \pm 1, \pm 2, \pm 3\}$ for every $\beta \vdash n$.

As $\gamma$ does not consist of odd distinct parts, it is then enough to prove that $\chi_{\gamma}^{\beta} \in\{0, \pm 1\}$ for every $\beta \vdash n$ not self conjugate with at least two $\gamma_{1}$ hooks, as then $\chi_{\gamma} \in\{0, \pm 1 / 2, \pm 1, \pm 3 / 2\}$, and so also $\chi_{\gamma} \in\{0, \pm 1\}$, for every irreducible character $\chi$ of $A_{n}$.

The next lemma is a generalization of Lemma 4.1 of [2].

Lemma 5.3. Let $\gamma=\left(\gamma_{1}, \ldots, \gamma_{r}\right)$ be a partition. Assume that $\left(\gamma_{2}, \ldots, \gamma_{r}\right)$ is a sign partition an that $\gamma_{2}+\ldots+\gamma_{r}<2 a$. If $\beta$ is a partition of $n$ for which $\chi_{\gamma}^{\beta} \notin\{0, \pm 1\}$ then $\beta$ has two $\gamma_{1}$-hooks. Also if $\delta$ is obtained from $\beta$ by removing a $\gamma_{1}$-hook then $\chi_{\left(\gamma_{2}, \ldots, \gamma_{r}\right)}^{\delta} \neq 0$. In particular each such $\delta$ has a $\gamma_{2}$-hook.

Proof. By assumption

$$
n=|\gamma|=\gamma_{1}+\gamma_{2}+\ldots+\gamma_{r}<3 a .
$$

It follows that any partition of $n$ has at most two $\gamma_{1}$-hooks. As

$$
\chi_{\gamma}^{\beta}=\sum_{(i, j): h_{i, j}^{\beta}=\gamma_{1}} \pm \chi_{\left(\gamma_{2}, \ldots, \gamma_{r}\right)}^{\beta \backslash R_{i, j}^{\beta}}
$$

and $\left(\gamma_{2}, \ldots, \gamma_{r}\right)$ is a sign partition by Theorem 1.3 of [2], so that $\chi_{\left(\gamma_{2}, \ldots, \gamma_{r}\right)}^{\beta \backslash R_{i, j}^{\beta}} \in$ $\{0, \pm 1\}$ for each $(i, j) \in[\beta]$ with $h_{i, j}^{\beta}=\gamma_{1}$, the lemma follows.

We will now prove that all remaining elements of $\overline{\text { Sign }}$ are $A_{n}$-sign partitions if they do not consists of odd distinct parts.

Theorem 5.4. If $a \geq 7$ then $(a, a-1,4,1)$ is an $A_{n}$-sign partition.

Proof. As $|(a, a-1,4,1)|=2 a+4<3 a$, so that any partition of $(a, a-$ $1,4,1)$ has at most two $a$-hooks, we only need to consider, from Lemma 5.2 , partitions of $2 a+4$ with $2 a$-hooks. Let $\beta$ be any such partition. Then the $a$ core of $\beta$ is a partition of 4 and so $\beta_{(a)} \in\{(4),(3,1),(2,2),(2,1,1),(1,1,1,1)\}$. As $\chi_{\rho}^{\lambda^{\prime}}= \pm \chi_{\rho}^{\lambda}$ and $\left(\lambda^{\prime}\right)_{(q)}=\left(\lambda_{(q)}\right)^{\prime}$ for $\lambda, \rho$ partitions and $q \geq 1$, we can assume that $\beta_{(a)} \in\{(4),(3,1),(2,2)\}$.

Assume first that $\beta_{(a)}=(4)$. From any $a$-core we can obtain exactly $a$ different partitions by adding an $a$-hook to it. In this case they are given by

$$
\{(a+4)\} \cup\left\{\left(4, i, 1^{a-i}\right): 1 \leq i \leq 4\right\} \cup\left\{\left(a-i, 5,1^{i-1}\right): 1 \leq i \leq a-5\right\} .
$$


If $\mu$ and $\nu$ are the partitions obtained from $\beta$ by removing an $a$-hook, we can assume from Lemma 5.3 that

$$
\mu, \nu \in\left\{(a+4),\left(4,1^{a}\right),\left(5,5,1^{a-6}\right),(a-1,5)\right\}
$$

as the other partition do not have $(a-1)$-hooks. From $a \geq 7$ and then

$$
\chi_{(a-1,4,1)}^{\left(4,1^{a}\right)}, \chi_{(a-1,4,1)}^{(a-1,5)}= \pm \chi_{(4,1)}^{(4,1)}=0
$$

we can further assume that $\mu, \nu \in\left\{(a+4),\left(5,5,1^{a-6}\right)\right\}$. Since $\mu \neq \nu$ and as we can recover $\beta$ from the $a$-cores and $a$-quotients of $\mu$ and $\nu$ (there exists a unique such partition $\beta$ ), we obtain that $\beta=\left(a+4,6,1^{a-6}\right)$ and then that

$\chi_{(a, a-1,4,1)}^{\beta}=-\chi_{(a-1,4,1)}^{\left(5,5,1^{a-6}\right)}+(-1)^{a-6} \chi_{(a-1,4,1)}^{(a+4)}=-(-1)^{a-6} \chi_{(4,1)}^{(5)}+(-1)^{a-6}=0$.

Assume next that $\beta_{(a)}=(3,1)$. In this case

$$
\begin{gathered}
\mu, \nu \in\left\{(a+3,1),(a, 4),\left(3,3,2,1^{a-4}\right),\left(3,2,2,1^{a-3}\right),\left(3,1^{a+1}\right)\right\} \\
\cup\left\{\left(a-i, 4,2,1^{i-2}\right): 2 \leq i \leq a-4\right\} .
\end{gathered}
$$

Again from Lemma 5.3 we can assume that $\mu$ and $\nu$ have an $(a-1)$-hook and so

$$
\mu, \nu \in\left\{(a+3,1),(a, 4),\left(3,2,2,1^{a-3}\right),\left(3,1^{a+1}\right),\left(4,4,2,1^{a-6}\right),(a-2,4,2)\right\} .
$$

Since

$$
\begin{aligned}
\chi_{(a-1,4,1)}^{(a+3,1)}, \chi_{(a-1,4,1)}^{\left(3,1^{a+1}\right)}, \chi_{(a-1,4,1)}^{\left(4,4,2,1^{a-6}\right)} & = \pm \chi_{(4,1)}^{(4,1)}=0 \\
\chi_{(a-1,4,1)}^{(a-2,4,2)} & =\chi_{(4,1)}^{(3,1,1)}=0
\end{aligned}
$$

we can assume that $\mu, \nu \in\left\{(a, 4),\left(3,2,2,1^{a-3}\right)\right\}$, that is $\beta=\left(a, 4,3,1^{a-3}\right)$. In this case

$$
\chi_{(a, a-1,4,1)}^{\beta}=(-1)^{a-3} \chi_{(a-1,4,1)}^{(a, 4)}+\chi_{(a-1,4,1)}^{\left(3,2,2,1^{a-3}\right)}=(-1)^{a-2} \chi_{(4,1)}^{(3,2)}+(-1)^{a-3} \chi_{(4,1)}^{(3,2)}=0 .
$$

Let now $\beta_{(a)}=(2,2)$. Then

$$
\begin{gathered}
\mu, \nu \in\left\{(a+2,2),(a+1,3),\left(2,2,2,1^{a-2}\right),\left(2,2,1^{a}\right)\right\} \\
\cup\left\{\left(a-i, 3,3,1^{i-2}\right): 2 \leq i \leq a-3\right\} .
\end{gathered}
$$

From $\mu$ and $\nu$ having an $(a-1)$-hook it follows

$$
\mu, \nu \in\left\{(a+2,2),\left(2,2,1^{a}\right),(a-2,3,3),\left(3,3,3,1^{a-5}\right)\right\} .
$$


As $(a+2,2)^{\prime}=\left(2,2,1^{a}\right)$ and $(a-2,3,3)^{\prime}=\left(3,3,3,1^{a-5}\right)$ and from $\beta$ being self-adjoint if $\mu=\nu^{\prime}$ (as the partitions obtained from $\beta^{\prime}$ by removing an $a$-hook are $\mu^{\prime}=\nu$ and $\nu^{\prime}=\mu$ in this case $)$ we can assume that $\mu \in\{(a+$ $\left.2,2),\left(2,2,1^{a}\right)\right\}$ and $\nu \in\left\{(a-2,3,3),\left(3,3,3,1^{a-5}\right)\right\}$. From $\chi_{\rho}^{\lambda}= \pm \chi_{\rho}^{\lambda^{\prime}}$, we can further assume that $\mu=(a+2,2)$. Then $\beta \in\{(a+2, a-1,3),(a+$ $\left.\left.2,4,3,1^{a-5}\right)\right\}$. From

$$
\begin{aligned}
\chi_{(a, a-1,4,1)}^{(a+2, a-1,3)} & =-\chi_{(a-1,4,1)}^{(a-2,3)}-\chi_{(a-1,4,1)}^{(a+2,2)}=-\chi_{(4,1)}^{(2,2,1)}-\chi_{(4,1)}^{(3,2)}=0, \\
\chi_{(a, a-1,4,1)}^{\left(a+2,4,3,1^{a-5}\right)} & =-\chi_{(a-1,4,1)}^{\left(3^{3}, 1^{a-5}\right)}+(-1)^{a-4} \chi_{(a-1,4,1)}^{(a+2,2)}=(-1+1)(-1)^{a-4} \chi_{(4,1)}^{(3,2)}=0
\end{aligned}
$$

it then follows that $(a, a-1,4,1)$ is an $A_{n}$-sign partition.

Theorem 5.5. If $a \geq 8$ then $(a, a-3,2,1,1)$ is an $A_{n}$-sign partition.

Proof. Since $|(a, a-3,2,1,1)|=2 a+1$ we only need to consider not selfadjoint $\beta \vdash 2 a+1$ with two $a$-hooks from Lemma 5.2. In this case $\beta_{(a)}=(1)$. Let $\mu, \nu$ be obtained from $\beta$ by removing these $a$-hooks. Then

$$
\mu, \nu \in\left\{(a+1),\left(1^{a+1}\right)\right\} \cup\left\{\left(a-i, 2,1^{i-1}\right): 1 \leq i \leq a-2\right\} .
$$

From Lemma 5.3 we can assume that $\mu$ and $\nu$ have an $(a-3)$-hook, so that

$$
\mu, \nu \in\left\{(a+1),\left(1^{a+1}\right),(a-1,2),(a-3,2,1,1),\left(4,2,1^{a-5}\right),\left(2^{2}, 1^{a-3}\right)\right\} .
$$

As $a-3>2$

$$
\chi_{(a-3,2,1,1)}^{(a-1,2)}, \chi_{(2,1,1)}^{\left(2^{2}, 1^{a-3}\right)}= \pm \chi_{(2,1,1)}^{(2,2)}=0
$$

we can further assume that

$$
\mu, \nu \in\left\{(a+1),\left(1^{a+1}\right),(a-3,2,1,1),\left(4,2,1^{a-5}\right)\right\} .
$$

From $(a+1)^{\prime}=\left(1^{a+1}\right)$ and $\left(a-3,2,1^{2}\right)^{\prime}=\left(4,2,1^{a-5}\right)$ we can, as in the previous theorem, assume that $\mu=(a+1)$ and $\nu \in\left\{\left(a-3,2,1^{2}\right),\left(4,2,1^{a-5}\right)\right\}$, so that $\beta \in\left\{(a+1, a-2,1,1),\left(a+1,5,1^{a-5}\right)\right\}$. From $a>5$ it follows

$$
\begin{aligned}
\chi_{(a, a-3,2,1,1)}^{(a+1, a-2,1,1)} & =\chi_{(a-3,2,1,1)}^{(a+1)}-\chi_{(a-3,2,1,1)}^{(a-3,2,1,1)}=1+\chi_{(2,1,1)}^{\left(1^{4}\right)}=0, \\
\chi_{(a, a-3,2,1,1)}^{\left(a+1,5,1^{a-5}\right)} & =(-1)^{a-5} \chi_{(a-3,2,1,1)}^{(a+1)}-\chi_{(a-3,2,1,1)}^{\left(4,2,1^{a-5}\right)}=(1-1)(-1)^{a-5} \chi_{(2,1,1)}^{(4)}=0
\end{aligned}
$$

it then follows from Lemma 5.2 that $(a, a-3,2,1,1)$ is an $A_{n}$-sign partition.

Theorem 5.6. If $a \geq 12$ then $(a, a-5,3,2,1)$ is an $A_{n}$-sign partition. 
Proof. As $|(a, a-5,3,2,1)|=2 a+1$ we only need to consider not self-adjoint $\beta \vdash 2 a+1$ with two $a$-hooks from Lemma 5.2. Also in this case $\beta_{(a)}=1$, so that if $\mu$ and $\nu$ are obtained from $\beta$ by removing an $a$-hook and they have an $(a-5)$-hook (as we can apply Lemma 5.3 ), we have that

$$
\begin{gathered}
\mu, \nu \in\left\{(a+1),\left(1^{a+1}\right),(a-1,2),(a-2,2,1),\left(a-3,2,1^{2}\right),\left(a-5,2,1^{4}\right),\right. \\
\left.\left(6,2,1^{a-7}\right),\left(4,2,1^{a-5}\right),\left(3,2,1^{a-4}\right),\left(2^{2}, 1^{a-3}\right)\right\} .
\end{gathered}
$$

From $a-5>4$ and then

$$
\begin{aligned}
& \chi_{(a-5,3,2,1)}^{(a-1,2)}, \chi_{(a-5,3,2,1)}^{\left(4,2,1^{a-5}\right)}= \pm \chi_{(3,2,1)}^{(4,2)}=0 \\
& \chi_{(a-5,3,2,1)}^{(a-2,1)}, \chi_{(a-5,3,2,1)}^{\left(3,2,1^{a-4}\right)}= \pm \chi_{(3,2,1)}^{(3,2,1)}=0 \\
& \chi_{\left(a-5,2,1^{2}\right)}^{\left(a-3,2,1^{2}\right)}, \chi_{(a-5,3,2,1)}^{\left(2^{2}, 1^{a-3}\right)}= \pm \chi_{(3,2,1)}^{(2,2,1,1)}=0
\end{aligned}
$$

we can assume that

$$
\mu, \nu \in\left\{(a+1),\left(1^{a+1}\right),\left(a-5,2,1^{4}\right),\left(6,2,1^{a-7}\right)\right\}
$$

and then again that $\mu=(a+1)$ and $\nu \in\left\{\left(a-5,2,1^{4}\right),\left(6,2,1^{a-7}\right)\right\}$, so $\beta \in\left\{\left(a+1, a-4,1^{4}\right),\left(a+1,7,1^{a-7}\right)\right\}$. From $a>11$ we have that

$$
\begin{aligned}
& \chi_{(a, a-5,3,2,1)}^{\left(a+1, a-4,1^{4}\right)}=\chi_{(a-5,3,2,1)}^{(a+1)}-\chi_{(a-5,3,2,1)}^{\left(a-5,2,1^{4}\right)}=1+\chi_{(3,2,1)}^{\left(1^{6}\right)}=0, \\
& \chi_{(a, a-5,3,2,1)}^{\left(a+1,7,1^{a-7}\right)}=(-1)^{a-7} \chi_{(a-5,3,2,1)}^{(a+1)}-\chi_{(a-5,3,2,1)}^{\left(6,2,1^{a-7}\right)}=(1-1)(-1)^{a-7} \chi_{(3,2,1)}^{(6)}=0 .
\end{aligned}
$$

In particular $(a, a-5,3,2,1)$ is an $A_{n}$-sign partition.

Theorem 5.7. If $b+1 \leq a \leq 2 b-2$ then $(a, b, a-b+1)$ is an $A_{n}$-sign partition if it does not consists of odd distinct parts.

Proof. From $b+1 \leq a \leq 2 b-2$ it follows that $a>b>a-b+1>1$. Again from Lemma 5.2 we only need to consider partitions $\beta$ of $|(a, b, a-b+1)|=2 a+1$ with two $a$-hooks which are not self-adjoint. From Lemma 5.3 in this case $\mu$ and $\nu$ have a $b$-hook, so that

$$
\begin{gathered}
\mu, \nu \in\left\{(a+1),\left(1^{a+1}\right)\right\} \cup\left\{\left(a-i, 2,1^{i-1}\right): 1 \leq i \leq a-b-2, i=a-b\right. \\
i=b-1 \text { or } b+1 \leq i \leq a-2\} .
\end{gathered}
$$

From $b>a-b+1$ it follows that any partition of $a+1$ has at most one $b$-hook, so that

$$
\chi_{(b, a-b+1)}^{\left(a-i, 2,1^{i-1}\right)}=\chi_{(a-b+1)}^{\left(a-b-i, 2,1^{i-1}\right)}=0
$$


for $1 \leq i \leq a-b-2$ and similarly for $b+1 \leq i \leq a-2$. As in the previous theorems we can assume that $\mu=(a+1)$ and $\nu \in\left\{\left(b, 2,1^{a-b-1}\right),(a-b+\right.$ $\left.\left.1,2,1^{b-2}\right)\right\}$, that is $\beta \in\left\{\left(a+1, b+1,1^{a-b-1}\right),\left(a+1, a-b+2,1^{b-2}\right)\right\}$. As

$\chi_{(a, b, a-b+1)}^{\left(a+1, b+1,1^{a-b-1}\right)}=(-1)^{a-b-1} \chi_{(b, a-b+1)}^{(a+1)}-\chi_{(b, a-b+1)}^{\left(b, 2,1^{a-b-1}\right)}=(-1)^{a-b-1}+\chi_{(a-b+1)}^{\left(1^{a-b+1}\right)}=0$,
$\chi_{(a, b, a-b+1)}^{\left(a+1, a-b+2,1^{b-2}\right)}=(-1)^{b-2} \chi_{(b, a-b+1)}^{(a+1)}-\chi_{(b, a-b+1)}^{\left(a-b+1,2,1^{b-2}\right)}=(-1)^{b-2}\left(1-\chi_{(a-b+1)}^{(a-b+1)}\right)=0$

we have that $(a, b, a-b+1)$ is an $A_{n}$-sign partition if it does not consists of odd distinct parts and $b+1 \leq a \leq 2 b-2$.

Theorem 5.8. If $b+2 \leq a \leq 2 b$ then $(a, b, a-b-1,1)$ is an $A_{n}$-sign partition if it does not consists of odd distinct parts.

Proof. Here $a>b>a-b-1 \geq 1$, as $b+2 \leq a \leq 2 b$. From Lemma 5.2 we only need to compute $\chi_{(a, b, a-b-1,1)}^{\beta}$ for $\beta \vdash 2 a$ not self-adjoint with two $a$-hook. If $\mu$ and $\nu$ are obtained from such a $\beta$ by removing an $a$-hook then $\mu$ and $\nu$ are $a$-hooks. From $a \leq 2 b$ it follows that $\mu$ and $\nu$ have at most one $b$-hook. If such a $b$-hook exists then

$$
\mu, \nu \in\left\{\left(a-i, 1^{i}\right): 0 \leq i \leq a-b-1 \text { or } b \leq i \leq a-1\right\} .
$$

For $1 \leq i \leq a-b-2$

$$
\chi_{(b, a-b-1,1)}^{\left(a-i, 1^{i}\right)}=\chi_{(a-b-1,1)}^{\left(a-i-b, 1^{i}\right)}=0
$$

and similarly for $b+1 \leq i \leq a-2$. So from Lemma 5.3 we can assume that

$$
\mu, \nu \in\left\{(a),\left(b+1,1^{a-b-1}\right),\left(a-b, 1^{b}\right),\left(1^{a}\right)\right\} .
$$

Also here we can assume $\mu=(a)$ and $\nu \in\left\{\left(b+1,1^{a-b-1}\right),\left(a-b, 1^{b}\right)\right\}$, that is $\beta \in\left\{\left(a, b+2,1^{a-b-2}\right),\left(a, a-b+1,1^{b-1}\right)\right\}$. From

$$
\begin{aligned}
& \chi_{(a, b, a-b-1,1)}^{\left(a, b+2,1^{a-b-2}\right)}=(-1)^{a-b-2} \chi_{(b, a-b-1,1)}^{(a)}-\chi_{(b, a-b-1,1)}^{\left(b+1,1^{a-b-1}\right)}=(-1)^{a-b}-\chi_{(a-b-1,1)}^{\left(1^{a-b}\right)}=0, \\
& \chi_{(a, b, a-b-1,1)}^{\left(a, a-b+1,1^{b-1}\right)}=(-1)^{b-1} \chi_{(b, a-b-1,1)}^{(a)}-\chi_{(b, a-b-1,1)}^{\left(a-b, 1^{b}\right)}=(-1)^{b-1}\left(1-\chi_{(a-b-1,1)}^{(a-b)}\right)=0
\end{aligned}
$$

it follows that $(a, b, a-b-1,1)$ is an $A_{n}$-sign partition if it does not consists of odd distinct parts.

\section{Acknowledgements}

The author thanks Christine Bessenrodt and Michael Cuntz for questions and discussion about sign conjugacy classes of $A_{n}$, which lead to writing this paper. 


\section{References}

[1] G. James, A. Kerber. The Representation Theory of the Symmetric Group. Addison-Wesley Publishing Company, 1981.

[2] L. Morotti. Sign conjugacy classes of the symmetric groups. Submitted.

[3] J. B. Olsson. Combinatorics and Representations of Finite Groups. Vorlesungen aus dem Fachbereich Mathematik der Univerität GH Essen, 1994. Heft 20.

[4] J. B. Olsson. Sign conjugacy classes in symmetric groups. Journal of Algebra 322 (2009) 2793-2800. 